\title{
Uma Revisão da Teoria Cinética em Física de Plasma para se Obter uma Forma Simplificada da Equação de Grad-Shafranov
}

An overview of kinetic theory in plasma physics to obtain a specific form of the Grad-Shafranov equation

\author{
M. Cristaldo-Oliveira ${ }^{1}$, A. Ojeda-González ${ }^{* 1 @}$ L. N. Santos ${ }^{1,2}$, \\ A. Prestes ${ }^{1}$, A. N. Laurindo Sousa ${ }^{1,30}$ \\ ${ }^{1}$ Universidade do Vale do Paraíba, Instituto de Pesquisa e Desenvolvimento, Laboratório de Física e Astronomia, \\ 12244-000, São José dos Campos, SP, Brasil. \\ ${ }^{2}$ Universidade Federal de Mato Grosso do Sul, Departamento de Matemática, 79200-000, Aquidauana, MS, Brasil. \\ ${ }^{3}$ Universidade Estadual do Maranhão, Centro de Estudos Superiores de Balsas, Departamento de Matemática, \\ 65800-000, Balsas, MA, Brasil.
}

Recebido em 18 de julho de 2020. Revisado em 08 de setembro de 2020. Aceito em 22 de setembro de 2020.

Esse trabalho tem por objetivo apresentar uma formulação teórica na área de física de plasma para obter uma forma simplificada da equação de Grad-Shafranov, em outras palavras, obter a lei de Ampère-Maxwell em função do potencial vetor magnético e da densidade de corrente. Buscamos entender melhor alguns fenômenos que ocorrem no plasma interplanetário. Outro objetivo é apresentar essa pesquisa de forma didática com a maior clareza e concisão possíveis. Para tanto, um desenvolvimento matemático se da como introdução ao trabalho, mostrando as equações que queremos deduzir, a cada seção vamos construindo uma base teórica em física de plasma, usando conceitos da termodinâmica e as leis universais da física, além de matemática, culminando na equação simplificada de Grad-Shafranov. Por fim, apresentamos uma das soluções mais conhecidas para equação de Grad-Shafranov, além de referências para quem quiser se aprofundar na pesquisa.

Palavras-chave: Magnetohidrodinâmica Ideal, Teoria Cinética, Lâmina de Corrente, Equação de GradShafranov.

This article aims to present a theoretical formulation in the area of plasma physics to obtain a specific form of the Grad-Shafranov equation, in other words, to obtain the Ampère-Maxwell law as a function of the magnetic vector potential and current density. We seek to better understand some phenomena that occur in interplanetary plasma. Another objective is to present this theme in a didactic way as clearly and concisely as possible. In particular, a mathematical development takes place as an introduction to the work, showing the equations we want to deduce, with each section we build a theoretical basis in plasma physics, using concepts of thermodynamics and the universal laws of physics, as well as mathematics, culminating in the Grad-Shafranov specific equation. Finally, we present one of the best known solutions for the Grad-Shafranov equation, as well as references for those who want to delve into the subject.

Keywords: Ideal Magnetohydrodynamics, Kinetic theory, Current sheet, Grad-Shafranov equation.

\section{Introdução}

O espaço interplanetário está quase totalmente imerso no plasma do vento solar e do meio interestelar [1]. Esse fluxo de partículas, denominado vento solar, encontra resistência próximo aos planetas devido a sua magnetosfera, seja ela do tipo induzida (Vênus e Marte) ou intrínseca (o restante dos planetas) 4. Plasma, por sua vez, é um tipo especial de gás altamente ionizado, composto primordialmente por elétrons e íons. Estas micropartículas que compõem o plasma podem interagir com os campos elétricos e magnéticos externos e internos. As interações Coulombianas entre partículas

\footnotetext{
* Endereço de correspondência: ojeda.gonzalez.a@gmail.com
}

carregadas, consideradas de longo alcance, levam aos movimentos coletivos do plasma. Esta substância e seu comportamento não é linear e nem tem um padrão predefinido, no entanto, a partir de simulações, via partículas, é possível interpretar fenômenos de larga escala e processos cinéticos que dependem do tempo, como por exemplo, instabilidades de ondas, difusão, aquecimento e aceleração de partículas em plasmas espaciais [5-7].

Também sabemos que o plasma, no meio interplanetário, está "congelado" ao campo magnético oriundo do Sol [8, 9]. O anterior é consequência do teorema de Alfvén 11 que é válido se $\nabla \times(\vec{E}+\vec{u} \times \vec{B})=0$, onde $\vec{u}$ é a

\footnotetext{
${ }_{1}$ Proposto pelo físico sueco Hannes O. G. Alfvén, laureado com o Nobel de Física de 1970.
} 
velocidade do plasma, e $\vec{E}$ e $\vec{B}$ são os campos elétricos e magnéticos respectivamente [10, 11]. O teorema afirma que, em um fluido perfeitamente condutor, o fluxo de campo magnético em um contorno fechado que se move com o plasma é constante. Este é o conceito de congelamento do plasma e permite que possamos visualizar as linhas de campo como estando congeladas no fluido. Um elemento de fluido que se move ao longo de um tubo de linhas de força, permanece em movimento com o tubo através do plasma.

O teorema de Alfvén geralmente é considerado válido se as seguintes condições forem satisfeitas:

a) a resistividade elétrica é muito pequena, ou seja, $\eta \approx 0$ (porém, a condutividade $\sigma=1 / \eta$ é quase infinita como acontece no meio interplanetário);

b) a densidade de corrente $(\vec{J})$ é pequena;

c) ambas escalas, espacial $L$ e temporal $t$ são muito grandes.

Embora o teorema de Alfvén seja válido na maioria das regiões do plasma que estudamos, ele não é verdadeiro nas lâminas de corrente, que são regiões estreitas de grande densidade de corrente [12]. A formação da própria lâmina de corrente é consequência do encontro de dois ou mais regimes de plasmas com suas respectivas configurações magnéticas, podendo interagir de tal forma que os plasmas se tornem interconectados, estabelecendo, assim, uma troca de energia, massa e momento que poderia levar às condições ideais para acontecer a reconexão magnética [11, 13, 14]. Sendo este, um dos fenômenos mais eficientes de troca de energia no interior do plasma em pequena e larga escala [15, 16].

Tendo em vista este cenário, a reconexão magnética que ocorre na magnetosfera terrestre é um importante fenômeno na ocorrência de sub-tempestades e tempestades geomagnéticas [17, 18], que por sua vez pode interferir em nossa tecnologia de satélites e redes elétricas [19]. Neste cenário, a equação apresentada neste artigo se torna importante para estudar a reconexão magnética e fazer reconstruções em modelos semiempíricos na magnetosfera e no vento solar [20 26].

Para começar o desenvolvimento deste trabalho se faz necessário enunciar as equações básicas do eletromagnetismo e conceitos da teoria cinética em física de plasma. Sendo assim, comecemos com a lei de AmpèreMaxwell, que diz que cargas em movimento produzem campo magnético. Ela pode ser transformada em duas novas equações: i) na equação de Grad-Shafranov (GS), quando a densidade de corrente é definida em função da primeira derivada do potencial vetor magnético e que não tem solução analítica [20, 22, 27]; ii) em uma forma simplificada da equação de GS, quando a densidade de corrente se escreve em função do exponencial do potencial vetor magnético que, neste caso, tem solução analítica [22, 24, 28, 32 .
A equação de GS como apresentada no primeiro caso do parágrafo anterior, deriva-se de um plasma considerado um fluído único, de alta condutividade, imerso num campo magnético, sem colisões e considerando que no interior do mesmo acontecem fenômenos de baixa frequência que permitem uma aproximação magnetostática [28, 29]. A condição de equilíbrio magnetostático define-se a partir da utilização da segunda lei de Newton, em uma amostra de plasma, no qual as forças gravitacional e magnética e o gradiente de pressão encontram-se com aceleração nula. A força gravitacional é desconsiderada por ser muito pequena em relação às outras duas forças. Porém, a equação de equilíbrio magnetostático, que da início ao desenvolvimento físico-matemático para a obtenção da equação de GS, é a seguinte:

$$
\nabla p=\vec{J} \times \vec{B}
$$

sendo que $p$ define a pressão cinética do plasma, $\vec{J}$ a densidade de corrente e $\vec{B}$ representa o vetor indução magnética. Pode-se encontrar o desenvolvimento detalhado de todo o formalismo matemático no trabalho de 22] e no apêndice de 31. Em todo o desenvolvimento algébrico que segue, utiliza-se o sistema de coordenadas Cartesianas, onde o vetor campo magnético é definido por $\vec{B}=B_{x}(x, z) \hat{i}+B_{y}(x, z) \hat{j}+B_{z}(x, z) \hat{k}$. Ou seja, considera-se o eixo $y$ invariante, o que leva fazer todas as possíveis visualizações das respetivas magnitudes do plasma no plano $x z$. Consequentemente a densidade de corrente define-se da seguinte maneira: $\vec{J}=J_{y}(x, z) \hat{j}$. A escolha do plano $x z$ justifica-se pelo fato que os modelos analíticos podem ser utilizados para realizar comparações com a magnetocauda da Terra quando se realiza um corte meridional com $x$ na linha Sol-Terra e $z$ no sentido sul magnético 33. Neste momento, fica necessário apresentar para o leitor a expressão da equação de GS:

$$
\frac{\partial^{2} A_{y}}{\partial x^{2}}+\frac{\partial^{2} A_{y}}{\partial z^{2}}=-\mu_{0} \frac{d}{d A_{y}}\left(p+\frac{B_{y}{ }^{2}}{2 \mu_{0}}\right),
$$

onde $A_{y}$ é a componente no eixo $y$ do vetor potencial magnético $\vec{A}$ e $\mu_{0}$ a permeabilidade magnética do vácuo. O termo da direita, no argumento da derivada, define a pressão transversal do plasma $\left(P_{t}\right)$ [20, 27, ou seja,

$$
P_{t}\left(A_{y}(x, z)\right)=p\left(A_{y}(x, z)\right)+\frac{B_{y}{ }^{2}\left(A_{y}(x, z)\right)}{2 \mu_{0}} .
$$

Existem casos muito específicos da expressão de $P_{t}$ para os quais é possível obter uma solução analítica de (2) 34 36. Um desses casos é a forma simplificada da equação de GS (referente ao item ii citado dois parágrafo acima) obtida por [28] a partir da teoria cinética de plasma, sendo essa equação o objeto de derivação deste trabalho. 
Ainda sem justificação física (será apresentada nas próximas seções) mas com apoio matemático e com o intuito de explicar ao leitor o porquê a nova equação é uma simplificação conveniente (ou seja, com solução analítica) de (2), define-se uma outra maneira de escrever (3):

$$
P_{t}=P_{t_{0}} \exp (-2 \Psi)
$$

no qual

$$
\Psi=-\frac{A_{y}}{L B_{0}}
$$

define o potencial vetor magnético normalizado, $B_{0}$ é o campo magnético assintótico, $L$ representa o comprimento de escala e

$$
P_{t_{0}}=\frac{B_{0}^{2}}{2 \mu_{0}}
$$

é a pressão transversal quando $A_{y}=0$ 37.

As expressões (3) são substituídas em 2 para obter a forma simplificada da equação de GS que tem a seguinte expressão:

$$
\frac{\partial^{2} \Psi}{\partial X^{2}}+\frac{\partial^{2} \Psi}{\partial Z^{2}}=e^{-2 \Psi}
$$

considerando novas variáveis que não possuem dimensões: $\frac{x}{L}=X$ e $\frac{z}{L}=Z$. A expressão matemática dada por (7) tem a forma de uma equação de Poisson.

O objetivo desde artigo é apresentar uma formulação teórica em física de plasma usando teoria cinética, necessária para se justificar fisicamente a escolha das equações (4)-(6) que permitem obter (7). Ou seja, a equação (7) em coordenadas cartesianas será construída passo a passo, para fins didáticos, mostrando caminhos para se entender melhor o tema.

O artigo está organizado da seguinte forma:

Seção 2 - Começamos definindo um espaço de fase e uma função de distribuição, ou seja, queremos a representação das variáveis dinâmicas do sistema para construção da equação de GS, além de uma função de distribuição e suas propriedades para representar a densidade de pontos apresentados no espaço de fase.

Seção 3 - Aqui definimos as grandezas macroscópicas do plasma como: momento, velocidade de fluxo, díade de fluxo de momento e tríade de fluxo total de energia. Usaremos isso para encontrar a equação de Boltzmann, como uma ferramenta adequada para análise de fenômenos de transporte que envolvem gradiente de temperatura e densidade. Nesta seção são feitas algumas hipóteses iniciais para se chegar na equação de Boltzmann mais adequada para equação de GS.

Seção 4 - Agora vamos encontrar a equação de Vlasov, para tanto usaremos as equações de Maxwell até encontrar uma expressão em função do vetor potencial magnético, também, uma forma analítica para a função de distribuição, levando em consideração tudo que já foi construído até aqui, usando os conceitos da termodinâmica para um sistema múltiplo em equilíbrio termodinâmico. Isso vai permitir calcular com eficiência a dinâmica da energia com o movimento gerado por ela.

Seção 5 - Utilizando todos as seções anteriores, como por exemplo que o plasma é frio, denso e não tem colisões. Deduziremos a equação de GS em sua forma simplificada devido a todas as restrições imposta à lei de Ampère-Maxwell.

Seção 6 - Por fim, nessa seção mostraremos uma possível solução para a equação, mas sem a intenção de aprofundamento nessa seção, já que o objetivo principal é deduzir a equação e comentar sua importância para a física.

Seção 7 - Na conclusão indicaremos as principais áreas de pesquisa e a relevância fundamental do assunto para a física e para nós seres humanos, que estamos inseridos nesse contexto. Entendemos que esse estudo pode ser ponta pé inicial para esse tema tão árduo, porém profícuo.

Durante o desenvolvimento das seções a seguir, utilizaremos a mesma notação implementada no Capítulo 5 do livro de [38]. É necessário mostrar para o leitor o raciocínio lógico seguido até obter a equação de Vlasov, para posteriormente deduzir a equação de GS em sua forma simplificada.

\section{Espaço de Fase e Função de Distribuição}

Num sistema de coordenadas Cartesianas, a posição do centro de massa das partículas é definida pelo vetor posição:

$$
\vec{r}=x \hat{i}+y \hat{j}+z \hat{k}
$$

cujo elemento de volume é $d^{3} r=d x d y d z$. Também, define-se a velocidade linear do centro de massa das partículas, a partir da primeira derivada em função do tempo da equação (8), obtendo-se:

$$
\vec{v}=v_{x} \hat{i}+v_{y} \hat{j}+v_{z} \hat{k}
$$

cujo elemento de volume é $d^{3} v=d v_{x} d v_{y} d v_{z}$. O elemento de volume permite definir uma função de distribuição ou distribuição de probabilidade, ou seja, a enumeração das probabilidades de todas as possíveis saídas de um determinado evento que aconteça no interior do plasma.

Continuamos por mencionar que o plasma possui um grande número de partículas com cargas elétricas positivas, negativas e neutras, mas macroscopicamente fica com carga nula. Para descrever as propriedades de um número grande de partículas definem-se quantas delas existem por unidade de volume num espaço de seis dimensões chamado de espaço de fase ou espaço- $\mu$ (do 
inglês, $\mu$-space), cujo elemento de volume representa-se por:

$$
d \vec{r} \cdot d \vec{v}=d x d y d z d v_{x} d v_{y} d v_{z}=d^{3} r d^{3} v .
$$

É importante ressaltar que do ponto de vista físico, para respeitar os critérios que definem um plasma, o elemento de volume $d^{3} r$ deve ser: $i$ ) grande quando comparado com o volume da esfera de Debye com raio $\lambda_{D}$ (comprimento de Debye), distância esta em que ocorre um equilíbrio entre a energia térmica das partículas que tende a perturbar a neutralidade elétrica e a energia potencial eletrostática, resultante de qualquer separação de carga o que tende a restaurar a neutralidade elétrica; ii) pequeno com relação à dimensão macroscópica do sistema, definido por L. Assim, $\lambda_{D}<r<L$ garante a neutralidade macroscópica do plasma e que no interior do elemento de volume exista um número significativo de partículas da espécie $\alpha$.

Em conclusão, as seis coordenadas do espaço de fase são $\left(x, y, z, v_{x}, v_{y}, v_{z}\right)$ e, segundo o livro de [38], a forma mais fácil e didática de representá-lo é num plano bidimensional como mostra a Figura 1 .

Após a definição do espaço de fase, precisa-se definir uma grandeza física que possibilite representar todas as partículas presentes no interior do plasma. Para isso, utiliza-se $N_{\alpha}$ como sendo a quantidade de partículas da espécie ou tipo $\alpha$ na posição $\vec{r}$, com velocidade $\vec{v}$ no tempo $t$. Sendo que, $d^{6} N_{\alpha}(\vec{r}, \vec{v}, t)$ é o número de partículas da espécie $\alpha$ que está no interior de um elemento de volume $d^{3} r$, com velocidade $\vec{v}$ dentro de $d^{3} v$ no instante t. E a função de distribuição das partículas da espécie $\alpha$ define-se pela seguinte expressão:

$$
f_{\alpha}(\vec{r}, \vec{v}, t)=\frac{d^{6} N_{\alpha}(\vec{r}, \vec{v}, t)}{d^{3} r d^{3} v},
$$

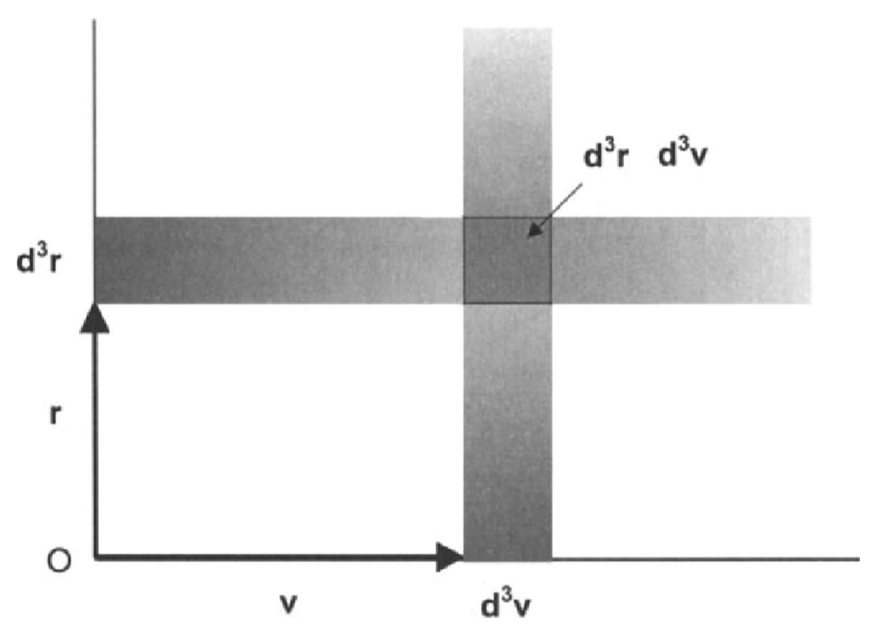

Figura 1: Representação do espaço de fase num plano bidimensional com eixos formados por $\vec{r}$ e $\vec{v}$. A representação permite visualizar um elemento de volume $d^{3} r d^{3} v$ importante para definir uma função de distribuição das partículas no interior do plasma [38]. para quantificar a densidade de pontos representativos no espaço de fase. Do ponto de vista matemático, para ter sentido físico, $f_{\alpha}(\vec{r}, \vec{v}, t)$ necessariamente precisará ser contínua, positiva e finita para todo instante $t$. Sendo que,

$$
\begin{aligned}
& \lim _{|\vec{v}| \rightarrow \infty} f_{\alpha}(\vec{r}, \vec{v}, t)=0 \\
& \int_{v} f_{\alpha}(\vec{r}, \vec{v}, t) d^{3} v=1 .
\end{aligned}
$$

A função de distribuição, também poderá ter as seguintes propriedades:

(i) Se $f_{\alpha}$ depende de $\vec{r}$ o plasma é não-homogêneo. Neste caso, na ausência de uma força externa no decorrer do tempo, as partículas interagem até um estado de equilíbrio térmico. No caso em que $f_{\alpha}$ não dependa da posição do vetor $\vec{r}$, o plasma considera-se homogêneo.

(ii) Se no plasma, em qualquer direção a velocidade for a mesma, será chamado de isotrópico, ou seja, $f_{\alpha}$ somente poderá depender da intensidade do vetor velocidade $v=|\vec{v}|$, caso contrário, se $f_{\alpha}$ depender da direção de $\vec{v}$, o plasma é anisotrópico.

(iii) Um plasma em equilíbrio térmico, por exemplo, é caracterizado por ser homogêneo, isotrópico e independente do tempo.

Uma vez que se conheça $f_{\alpha}(\vec{r}, \vec{v}, t)$, será possível deduzir todas as magnitudes ou grandezas macroscópicas de interesse físico da espécie $\alpha$, presentes no plasma, que será o objetivo da próxima seção.

\section{Grandezas Macroscópicas do Plasma}

A primeira e mais simples grandeza física que se pode definir, a partir da função de distribuição $f_{\alpha}(\vec{r}, \vec{v}, t)$, é a densidade de partículas com dependência espacial, representada por $n_{\alpha}(\vec{r}, t)$ e caraterizada por ser independente da velocidade. Ela representa o número de partículas da espécie $\alpha$, por unidade de volume, podendo ser obtida integrando $d^{6} N_{\alpha}(\vec{r}, \vec{v}, t)$ sobre todo o espaço de velocidades e dividindo o resultado pelo elemento de volume $d^{3} r$, como segue:

$$
\begin{aligned}
n_{\alpha}(\vec{r}, t) & =\frac{1}{d^{3} r} \int_{v} d^{6} N_{\alpha}(\vec{r}, \vec{v}, t) \\
& =\int_{v} \frac{d^{6} N_{\alpha}(\vec{r}, \vec{v}, t)}{d^{3} r} \\
& =\int_{v} f_{\alpha}(\vec{r}, \vec{v}, t) d^{3} v .
\end{aligned}
$$

A densidade de partículas também é chamada de momento de ordem zero.

A segunda grandeza física é a Velocidade de Fluxo Macroscópica, também chamada de Velocidade Média 
do Plasma. Esta grandeza é representada por $\vec{v}_{\alpha}(\vec{r}, t)$ que é a velocidade de fluxo macroscópico do plasma de partículas da espécie $\alpha$.

A fórmula fica definida pela seguinte expressão:

$$
\begin{aligned}
\vec{v}_{\alpha}(\vec{r}, t) & =\frac{1}{n_{\alpha}(\vec{r}, t) d^{3} r} \int_{v} \vec{v} d^{6} N_{\alpha}(\vec{r}, \vec{v}, t) \\
& =\frac{1}{n_{\alpha}(\vec{r}, t)} \int_{v} \vec{v} f_{\alpha}(\vec{r}, \vec{v}, t) d^{3} v .
\end{aligned}
$$

A Velocidade Média do Plasma também é conhecida como momento de primeira ordem.

Seguindo com o raciocínio anterior, é possível obter outras variáveis macroscópicas para a díade de fluxo de momento $\Pi_{\alpha}(\vec{r}, t)$ (momento de segunda ordem) e para a tríade de fluxo total de energia $\xi_{\alpha}(\vec{r}, t)$ (momento de terceira ordem). Em [38], define-se uma fórmula genérica para se obter qualquer momento de ordem $N$ como segue:

$$
M_{\alpha i j \cdots k}^{(N)}(\vec{r}, t)=\int_{v} v_{i} v_{j} \cdots v_{k} f_{\alpha}(\vec{r}, \vec{v}, t) d^{3} v .
$$

Para as deduções físicas apresentadas nas seções posteriores, são necessários somente os momentos $n_{\alpha}(\vec{r}, t)$ e $\vec{v}_{\alpha}(\vec{r}, t)$. Com as definições anteriores tem-se todos os ingredientes necessários para definir a equação de Boltzmann.

\subsection{Equação de Boltzmann}

Para continuar com o raciocínio, é preciso somente dar atenção a uma amostra de partículas idênticas da espécie $\alpha$ dentro do elemento de volume $d^{3} r d^{3} v$ no espaço de fase, no tempo $t$. Considera-se que as partículas não interagem entre si, desconsiderando, assim, as interações de curto-alcance responsáveis pelas colisões. Desta maneira, consideram-se somente as interações coulombianas de longo alcance, responsáveis pelo comportamento coletivo do plasma, e que serão descritas pela equação de Boltzmann apresentada mais adiante.

No tempo $t$, as partículas dentro do elemento de volume $d^{3} r d^{3} v$ são descritas pela função de distribuição $f_{\alpha}(\vec{r}, \vec{v}, t)$. Considerando uma pequena variação temporal $d t$, o sistema de partículas evolui no espaço de fase para um novo elemento de volume $d^{3} r^{\prime} d^{3} v^{\prime}$ com novas coordenadas, isto é, de $(\vec{r}, \vec{v}, t)$ para $\left(\vec{r}^{\prime}, \vec{v}^{\prime}, t+d t\right)$. No novo elemento de volume do espaço de fase, $f_{\alpha}$ será descrita em função das novas coordenadas, sendo então definida por $f_{\alpha}\left(\vec{r}^{\prime}, \vec{v}^{\prime}, t+d t\right)$. Como foi considerado a ausência de interações internas, pode-se supor que a transição das partículas, no espaço de fase de um elemento de volume para outro, foi possível pela presença de uma força externa $\vec{F}$. Assim sendo, a variação do vetor posição segue como:

$$
\vec{r}^{\prime}(t+d t)=\vec{r}(t)+\vec{v} d t
$$

e a variação do vetor velocidade tem a forma:

$$
\vec{v}^{\prime}(t+d t)=\vec{v}(t)+\vec{a} d t
$$

sendo o vetor $\vec{a}$, a aceleração da partícula.

Pela segunda lei de Newton temos:

$$
\vec{a}=\frac{\vec{F}}{m_{\alpha}}
$$

sendo $m_{\alpha}$ a massa da partícula da espécie $\alpha$. Considerando que não há alterações na quantidade e tipo de partículas de uma determinada espécie, nos instantes $t$ e $t+d t$ e que não há colisões entre elas, segue:

$$
d^{6} N_{\alpha}\left(\vec{r}^{\prime}, \vec{v}^{\prime}, t+d t\right)=d^{6} N_{\alpha}(\vec{r}, \vec{v}, t) .
$$

Sendo necessário que $d^{3} r^{\prime} d^{3} v^{\prime}=|J| d^{3} r d^{3} v$, no qual $|J|=1$ é o determinante do Jacobiano (ver demonstração no capítulo 5 do livro de [38]), para assim obter a relação da função de distribuição entre os elementos de volume:

$$
f_{\alpha}\left(\vec{r}^{\prime}, \vec{v}^{\prime}, t+d t\right) d^{3} r^{\prime} d^{3} v^{\prime}=f_{\alpha}(\vec{r}, \vec{v}, t) d^{3} r d^{3} v .
$$

Da equação 21 segue que,

$$
f_{\alpha}\left(\vec{r}^{\prime}, \vec{v}^{\prime}, t+d t\right) d^{3} r^{\prime} d^{3} v^{\prime}-f_{\alpha}(\vec{r}, \vec{v}, t) d^{3} r d^{3} v=0 .
$$

Utilizando a relação $d^{3} r^{\prime} d^{3} v^{\prime}=d^{3} r d^{3} v$, pode-se deixar em evidência o termo $d^{3} r d^{3} v$ na equação 22 :

$$
\left[f_{\alpha}\left(\vec{r}^{\prime}, \vec{v}^{\prime}, t+d t\right)-f_{\alpha}(\vec{r}, \vec{v}, t)\right] d^{3} r d^{3} v=0 .
$$

Para prosseguir com o desenvolvimento matemático da equação 23, primeiramente será necessário fazer uma expansão em série de Taylor de $f_{\alpha}\left(\vec{r}^{\prime}, \vec{v}^{\prime}, t+d t\right)$ até primeira ordem no tempo $t$, ou seja, os termos a partir de $(d t)^{2}$ serão desconsiderados. A expansão em série de Taylor até primeira ordem segue que:

$$
f_{\alpha}\left(\vec{r}^{\prime}, \vec{v}^{\prime}, t+d t\right)=f_{\alpha}(\vec{r}, \vec{v}, t)+\left[\frac{d f_{\alpha}(\vec{r}, \vec{v}, t)}{d t}\right] d t .
$$

Utilizando a regra da cadeia no segundo termo da direita da equação (24), se obtém,

$$
\begin{aligned}
f_{\alpha}\left(\vec{r}^{\prime}, \vec{v}^{\prime}, t+d t\right)= & f_{\alpha}(\vec{r}, \vec{v}, t) \\
& +\left[\frac{\partial f_{\alpha}}{\partial t}+\frac{\partial f_{\alpha}}{\partial \vec{r}} \frac{d \vec{r}}{d t}+\frac{\partial f_{\alpha}}{\partial \vec{v}} \frac{d \vec{v}}{d t}\right] d t .
\end{aligned}
$$

A equação 25 escreve-se em função das componentes dos vetores $\vec{r}$ e $\vec{v}$ como segue:

$$
\begin{aligned}
f_{\alpha}\left(\vec{r}^{\prime}, \vec{v}^{\prime}, t+d t\right) & =f_{\alpha}(\vec{r}, \vec{v}, t)+\left[\frac{\partial f_{\alpha}}{\partial t}+\left(v_{x} \frac{\partial f_{\alpha}}{\partial x}+v_{y} \frac{\partial f_{\alpha}}{\partial y}+v_{z} \frac{\partial f_{\alpha}}{\partial z}\right)\right. \\
& \left.+\left(a_{x} \frac{\partial f_{\alpha}}{\partial v_{x}}+a_{y} \frac{\partial f_{\alpha}}{\partial v_{y}}+a_{z} \frac{\partial f_{\alpha}}{\partial v_{z}}\right)\right] d t
\end{aligned}
$$


Substituindo 26 em 23 ,

$$
\begin{aligned}
{\left[f_{\alpha}\right.} & +\left[\frac{\partial f_{\alpha}}{\partial t}+\left(v_{x} \frac{\partial f_{\alpha}}{\partial x}+v_{y} \frac{\partial f_{\alpha}}{\partial y}+v_{z} \frac{\partial f_{\alpha}}{\partial z}\right)\right. \\
& \left.\left.+\left(a_{x} \frac{\partial f_{\alpha}}{\partial v_{x}}+a_{y} \frac{\partial f_{\alpha}}{\partial v_{y}}+a_{z} \frac{\partial f_{\alpha}}{\partial v_{z}}\right)\right] d t-f_{\alpha}\right] \\
& \times d^{3} r d^{3} v=0 .
\end{aligned}
$$

Note que os termos envolvendo $f_{\alpha}$ se cancelam e que também pode-se reescrever toda a equação em função do operador nabla tanto para $\vec{r}$,

$$
\nabla=\hat{i} \frac{\partial}{\partial x}+\hat{j} \frac{\partial}{\partial y}+\hat{k} \frac{\partial}{\partial z}
$$

como para $\vec{v}$

$$
\nabla_{\vec{v}}=\hat{i} \frac{\partial}{\partial v_{x}}+\hat{j} \frac{\partial}{\partial v_{y}}+\hat{k} \frac{\partial}{\partial v_{z}} .
$$

Resultando em:

$$
\frac{\partial}{\partial t} f_{\alpha}(\vec{r}, \vec{v}, t)+\vec{v} \cdot \nabla f_{\alpha}(\vec{r}, \vec{v}, t)+\vec{a} \cdot \nabla_{\vec{v}} f_{\alpha}(\vec{r}, \vec{v}, t)=0 .
$$

A equação (30) é chamada equação de Boltzmann na ausência de colisões. Lembrando que a derivada total com respeito ao tempo no espaço de fase é dado pela equação

$$
\frac{D}{D t}=\frac{\partial}{\partial t}+\vec{v} \cdot \nabla+\vec{a} \cdot \nabla_{\vec{v}},
$$

pode-se reescrever a equação de Boltzmann como:

$$
\frac{D}{D t} f_{\alpha}(\vec{r}, \vec{v}, t)=0
$$

A equação anterior representa um estado de conservação da densidade de pontos, $f_{\alpha}(\vec{r}, \vec{v}, t)$, representativos no espaço de fase. Nesta situação, o teorema de Liouville pode ser enunciado. Este teorema diz que a quantidade de partículas num determinado instante de tempo $t$, que estejam contidas no elemento de volume $d^{3} r d^{3} v$, continuam estando num instante posterior $t+d t$ em $d^{3} r^{\prime} d^{3} v^{\prime}$. Isso implica que o Jacobiano $|J|$ entre os elementos de volume no espaço de fase, definido por $d^{3} r^{\prime} d^{3} v^{\prime}=|J| d^{3} r d^{3} v$, seja 1 como foi mencionado no começo da seção. Do ponto de vista físico, o teorema significa que, tanto as colisões, assim como a emissão de energia por radiação e os processos de produção e perda de partículas, tornam-se desprezíveis.

Considerando-se colisões entre as partículas e suas consequências decorrentes, tem-se $d^{3} r^{\prime} d^{3} v^{\prime} \neq d^{3} r d^{3} v$, pois no decorrer do tempo $d t$, partículas podem ser perdidas ou ganhas nos respectivos elementos de volume. Isto implica que o teorema de Liouville não é válido para um sistema em que colisões entre as partículas estão presentes. Portanto, a equação de Boltzmann com colisões ficará:

$$
\frac{D}{D t} f_{\alpha}(\vec{r}, \vec{v}, t)=\left(\frac{\delta f_{\alpha}}{\delta t}\right)_{\text {coll }},
$$

onde $\left(\frac{\delta f_{\alpha}}{\delta t}\right)_{\text {coll }}$ é o termo que denota colisões.
Existem alguns modelos para o termo de colisões, de acordo com [38, a saber:

- Modelo de Krook ou relaxation model (o mais simples);

- Boltzman Collision Integral;

- Fokker-Planck collision term.

A equação de Boltzmann sem colisões se escreve em função da força resultante que atua sobre o plasma para obter a equação de Vlasov.

\section{Equação de Vlasov}

Retornando à equação de Boltzmann sem colisões e à força total que pode ser utilizada na expressão da aceleração, conforme a segunda lei de Newton, a equação (30) pode ser reescrita como:

$\frac{\partial}{\partial t} f_{\alpha}(\vec{r}, \vec{v}, t)+\vec{v} \cdot \nabla f_{\alpha}(\vec{r}, \vec{v}, t)+\frac{\vec{F}_{\text {total }}}{m_{\alpha}} \cdot \nabla_{\vec{v}} f_{\alpha}(\vec{r}, \vec{v}, t)=0$,

onde $\vec{F}_{\text {total }}$ é a soma das forças externas com as forças internas, tal que essas forças internas decorrem dos campos eletromagnéticos internos $\vec{E}_{i}$ e $\vec{B}_{i}$, consequentes do movimento entre as partículas e que formam a força de Lorentz.

Substituindo a força externa e a força de Lorentz em (34), teremos que:

$\frac{\partial f_{\alpha}}{\partial t}+\vec{v} \cdot \nabla f_{\alpha}+\frac{1}{m_{\alpha}}\left[\vec{F}_{e x t}+q_{\alpha}\left(\vec{E}_{i}+\vec{v} \times \vec{B}_{i}\right)\right] \cdot \nabla_{\vec{v}} f_{\alpha}=0$,

chamada de equação de Vlasov, sendo que os campos $\vec{E}_{i}$ e $\vec{B}_{i}$ devem satisfazer as leis de Maxwell. Com o intuito de simplificar a notação, de agora em diante utilizaremos $\vec{E}_{i}=\vec{E}$ e $\vec{B}_{i}=\vec{B}$.

Para resolver (35) necessita-se da densidade de carga $\rho(\vec{r}, t)$, da densidade de corrente $\vec{J}(\vec{r}, t)$ e das equações de Maxwell, todas elas devem ser resolvidas de uma maneira auto-consistente.

\subsection{Potencial vetor magnético nas equações de Maxwell}

Para poder definir uma forma analítica de $f_{\alpha}(\vec{r}, \vec{v}, t)$, precisa-se introduzir o uso do potencial vetor magnético nas equações de Maxwell. Umas das equações de Maxwell diz que $\nabla \cdot \vec{B}=0$, e isto nos permite definir um potencial vetor magnético $\vec{A}$,

$$
\vec{B}=\nabla \times \vec{A} \Longrightarrow \nabla \cdot(\nabla \times \vec{A})=0,
$$

e pela lei de Faraday temos:

$$
\nabla \times \vec{E}+\frac{\partial \vec{B}}{\partial t}=0,
$$

ou seja:

$$
\nabla \times\left(\vec{E}+\frac{\partial \vec{A}}{\partial t}\right)=0
$$


Considerando a identidade matemática $\nabla \times(\nabla C(r))=$ 0 , pode-se definir o potencial $\vec{E}+\partial \vec{A} / \partial t$ como sendo o gradiente de uma função escalar, sendo ela o potencial eletrostático $\phi(\vec{r})$, assim:

$$
\vec{E}+\frac{\partial \vec{A}}{\partial t}=-\nabla \phi
$$

sendo que, o campo elétrico fica definido em função do potencial eletrostático e do potencial vetor magnético como segue:

$$
\vec{E}=-\nabla \phi-\frac{\partial \vec{A}}{\partial t}
$$

Novamente, das equações de Maxwell teremos que:

$$
\nabla \times \vec{B}=\mu_{0}\left(\vec{J}+\epsilon_{0} \frac{\partial \vec{E}}{\partial t}\right)
$$

e utilizando (36),

$$
\nabla \times \vec{B}=\nabla \times(\nabla \times \vec{A})=\mu_{0}\left(\vec{J}+\epsilon_{0} \frac{\partial \vec{E}}{\partial t}\right) .
$$

Usando uma identidade vetorial, teremos:

$$
\nabla \times(\nabla \times \vec{A})=\nabla(\nabla \cdot \vec{A})-\nabla^{2} \vec{A}=\mu_{0}\left(\vec{J}+\epsilon_{0} \frac{\partial \vec{E}}{\partial t}\right)
$$

$\mathrm{Na}$ análise que segue, considera-se um sistema 2.5-D em que se analisa as variações de uma quantidade física em apenas uma direção, mas esta quantidade pode depender das outras duas variáveis do sistema em estudo. Ou seja, considera-se o eixo $y$ invariante, e nenhuma das grandezas físicas envolvidas no problema dependerão dele. As respectivas derivadas parciais serão nulas nesta direção. A invariância em um dos eixos de coordenada Cartesiana é uma condição imposta para reduzir a análise matemática do problema, que vai possibilitar obter uma forma simplificada da equação de GS. Uma explicação detalhada das implicações físicas da condição $\partial / \partial y=0$ foi discutida no trabalho [39]. Como consequência do eixo invariante e juntando 42 com 43 se obtém que:

$$
\nabla \times \vec{B}=-\nabla^{2} \vec{A}=\mu_{0}\left(\vec{J}+\epsilon_{0} \frac{\partial \vec{E}}{\partial t}\right) .
$$

A implicação geométrica da invariância em y é que as grandezas macroscópicas do sistema somente dependerão de $x$ e $z$. Consequentemente, $\vec{E}$ e $\vec{J}$ estarão na direção $y$. Poderíamos escrever a equação (44) em suas três componentes, mas como foi dito no final da Seção 1, adotamos um sistema de coordenadas que futuramente, talvez, possibilite fazer comparações com um corte meridional da magnetocauda [28, 33]. Sendo assim, trabalha-se somente com a componente y:

$$
\left(\frac{\partial^{2} A_{y}(x, z)}{\partial x^{2}}+\frac{\partial^{2} A_{y}(x, z)}{\partial z^{2}}\right)=-\mu_{0}\left(J_{y}+\epsilon_{0} \frac{\partial E_{y}}{\partial t}\right),
$$

O resultado é a equação generalizada de AmpèreMaxwell:

$$
\nabla^{2} A_{y}=-\mu_{0}\left(J_{y}+\epsilon_{0} \frac{\partial E_{y}}{\partial t}\right)
$$

que se utiliza como ponto de partida para obter a equação de GS. Em uma situação magnetostática $(\partial / \partial t=0)$, a equação (46) possui a mesma forma que a equação de Poisson da eletrostática, por analogia, concluímos que $A_{y}$ pode ser calculado mediante a seguinte equação:

$$
A_{y}(\vec{r})=\frac{\mu_{0}}{4 \pi} \iiint_{V^{\prime}} \frac{J_{y}\left(\vec{r}^{\prime}\right) d V^{\prime}}{\left|\vec{r}-\vec{r}^{\prime}\right|}
$$

Do ponto de vista geométrico os vetores $\vec{A}$ e $\vec{J}$ estão orientados na mesma direção.

\subsection{Forma analítica da função de distribuição}

Para obter uma expressão de $A_{y}$ em 47) que seja solução de 46 é necessário encontrar uma expressão de $J_{y}$. O desenvolvimento teórico que segue irá transformar o termo da direita de (46), ou seja, $J_{y}$ será expressa como uma função exponencial. Desta maneira, 46 se tornará semelhante à equação encontrada por Liouville em 1853 [37, 40], em que será possível encontrar, em teoria, infinitas soluções [30. Fisicamente falando, a maneira mais adequada de fazer o raciocínio anterior é resolvendo o sistema de equações de Vlasov-Maxwell escolhendo uma função de distribuição que se ajuste às propriedades do plasma em estudo.

Neste trabalho, considera-se um plasma numa situação ideal em equilíbrio magnetostático. Sendo este um sistema 2,5-D, ou seja, existe componente do campo em $y$ mas as grandezas físicas envolvidas no problema somente dependem das variáveis $x$ e $z$ 39]. Nesta configuração, a corrente elétrica, o campo elétrico e o vetor potencial estão predominantemente na direção de $y$. $\mathrm{O}$ interessante deste problema é que a solução do sistema de equações de Vlasov-Maxwell, nesta situação ideal, terá como resultado a obtenção de lâminas de corrente bidimensional transversal ao plano $x z$. Sendo esta uma situação que permitirá fazer modelos analíticos de lâminas de corrente para realizar estudos na magnetocauda, como feito por [28, 33, 41,43].

Para iniciar o desenvolvimento do problema, reescrevemos 14 e 15 para definir as densidades de carga e de corrente como segue:

$$
\begin{aligned}
\rho(x, z, t=0) & =\sum_{\alpha} q_{\alpha} n_{\alpha}(x, z) \\
& =\sum_{\alpha} q_{\alpha}\left[\int_{v} f_{\alpha}(x, z, \vec{v}) d^{3} v\right]
\end{aligned}
$$


e

$$
\begin{aligned}
\vec{J}(x, z, t=0) & =\sum_{\alpha} q_{\alpha} n_{\alpha}(x, z) \vec{v}_{\alpha}(x, z) \\
& =\sum_{\alpha} q_{\alpha}\left[\int_{v} \vec{v} f_{\alpha}(x, z, \vec{v}) d^{3} v\right] .
\end{aligned}
$$

O plasma considerado neste problema é pouco denso e frio e sem colisões. Sendo assim, se considera nula a força externa na equação de Vlasov (ver (35)). Supõe-se para efeito de cálculo que não há variações temporais, isto implica que $\frac{\partial f_{\alpha}}{\partial t}=0$. Com as considerações anteriores, o sistema de equações de Vlasov-Maxwell é formado por três equações, isto é,

$$
\begin{gathered}
\vec{v} \cdot \nabla f_{\alpha}+\frac{1}{m_{\alpha}}\left[q_{\alpha}(\vec{E}+\vec{v} \times \vec{B})\right] \cdot \nabla_{\vec{v}} f_{\alpha}=0, \\
\nabla \cdot \vec{E}=\frac{1}{\epsilon_{0}} \sum_{\alpha} q_{\alpha}\left[\int_{v} f_{\alpha}(x, z, \vec{v}) d^{3} v\right],
\end{gathered}
$$

$\mathrm{e}$

$$
\nabla \times \vec{B}=\mu_{0} \sum_{\alpha} q_{\alpha}\left[\int_{v} \vec{v} f_{\alpha}(x, z, \vec{v}) d^{3} v\right] .
$$

Em termodinâmica, num sistema com múltiplos estados em equilíbrio termodinâmico, é utilizado um fator de ponderação para determinar a probabilidade relativa de um estado $i$. O fator de ponderação recebe o nome de fator de Boltzmann, que em si mesmo não é uma probabilidade, porque não está normalizado. Dividindo o fator de Boltzmann pela probabilidade de todos os estados possíveis do sistema, se obtém a distribuição de Boltzmann. Esse fator relaciona a energia do estado $i\left(\Omega_{i}\right)$ com a energia térmica do sistema por meio da expressão $\exp \left[-\Omega_{i} / k_{B} T_{i}\right]$, sendo $k_{B}$ a constante de Boltzmann e $T_{i}$ a temperatura. Utilizando o fator de Boltzmann podese desenvolver a estatística de Maxwell-Boltzmann, a qual considera que, num plasma em equilibro termodinâmico, as partículas da espécie são caraterizadas por uma função contínua de velocidades dada pela seguinte expressão:

$$
\begin{aligned}
f_{\alpha}\left(v_{x}, v_{y}, v_{z}\right) & =f_{\alpha}(\vec{v}) \\
& =\left(\frac{m_{\alpha}}{2 \pi k_{B} T_{\alpha}}\right)^{3 / 2} \exp \left[-\frac{m_{\alpha}}{2 k_{B} T_{\alpha}} v^{2}\right] .
\end{aligned}
$$

Sendo que a energia do estado $i$ do fator de Boltzmann é a energia cinética total das partículas $\left(K_{\alpha}=\left(m_{\alpha} v^{2}\right) / 2\right)$. Fica evidente que a equação 53 satisfaz a condição de normalização, ou seja:

$$
\int_{v} f_{\alpha}(\vec{v}) d^{3} v=1
$$

A equação (53), assim como se apresenta, depende apenas do módulo da velocidade. Não depende da posição $\vec{r}$, que poderá ser introduzida usando o fator de Boltzmann para definir uma função de distribuição espacial da densidade de partículas $n_{\alpha}(x, z)$. Isto é,

$$
n_{\alpha}(x, z)=n_{0_{\alpha}} \exp \left[-\frac{V_{\alpha}(x, z)}{k_{B} T_{\alpha}}\right],
$$

em que $n_{0_{\alpha}}$ é a densidade total de partículas no interior de uma esfera de raio $r$. Note que o numerador do argumento da exponencial no fator de Boltzmann, será uma função que depende apenas da energia potencial total das partículas $\left(V_{\alpha}(x, z)\right)$.

Voltando ao problema inicial representado pela função de distribuição $f_{\alpha}(x, z, \vec{v})$, pode-se utilizar um método de separação de variáveis como segue:

$$
f_{\alpha}(x, z, \vec{v})=n_{\alpha}(x, z) f_{\alpha}(\vec{v}) .
$$

Segundo [4] e posteriormente usado nos trabalhos de [28 30], para se obter uma expressão final de (56), ainda acrescentam-se duas considerações: $i$ ) os íons positivos se movimentam com uma velocidade de deriva $U_{i}$ no sentido positivo do eixo $y$ e os elétrons com velocidade $U_{e}$ no sentido oposto, mas para manter a mesma notação, ela aparecerá nas equações como $\left.U_{\alpha} ; i i\right)$ a função de distribuição para satisfazer o sistema de equações de Vlasov-Maxwell deverá depender de constantes de movimento, ou seja, da Hamiltoniana $(\mathrm{H})$ e do momento Canônico $\vec{p}$. Logo, a dependência funcional em 56 passará a ser $f_{\alpha}(x, z, \vec{v})=f_{\alpha}\left(H, p_{y}\right)$.

Fazendo uma revisão da Mecânica Clássica, definem-se as coordenadas canônicas como sendo $p_{i}$ e $q_{i}$. Estas coordenadas estão relacionadas por meio da Lagrangeana $L\left(q_{i}, q_{i}^{\prime}, t\right)$, sendo $p_{i}=\partial L / \partial q_{i}^{\prime}$. Define-se a Hamiltoniana a partir da equação $H=\sum_{i}\left[p_{i} q_{i}^{\prime}\right]-L$.

Considerando a força de Lorentz:

$$
\vec{F}_{\alpha}(\vec{r}, \vec{v})=q_{\alpha}[\vec{E}(\vec{r})+\vec{v} \times \vec{B}(\vec{r})],
$$

a Lagrangeana relacionada com esta força é

$$
L_{\alpha}(\vec{r}, \vec{v})=\frac{1}{2} m_{\alpha} \vec{v}^{2}+q_{\alpha} \vec{v} \cdot \vec{A}(\vec{r})-q_{\alpha} \phi(\vec{r}),
$$

na qual $\phi(\vec{r})$ é o potencial elétrico. $\mathrm{O}$ momento canônico desta Lagrangeana define-se como

$$
\vec{p}_{\alpha}(\vec{r}, \vec{v})=m_{\alpha} \vec{v}+q_{\alpha} \vec{A}(\vec{r}) .
$$

Logo, a partir de 58 e 59 , considerando a velocidade de deriva $\left(U_{\alpha}\right)$ em $y$, que surge como consequência da geometria aqui adotada, pois terá um gradiente de campo magnético $\left(\nabla B_{y}\right)$ sempre contido no plano $x z \mathrm{e}$ majoritariamente direcionado ao longo de $z$, o resultado de (56) ficará

$$
\begin{aligned}
& f_{\alpha}(x, z, \vec{v}) \\
& =n_{0_{\alpha}} \exp \left[-\frac{q_{\alpha} \phi(\vec{r})-q_{\alpha} U_{\alpha} A_{y}(x, z)}{k_{B} T_{\alpha}}\right]\left(\frac{m_{\alpha}}{2 \pi k_{B} T_{\alpha}}\right)^{3 / 2} \\
& \quad \times \exp \left[-\frac{m_{\alpha}}{2 k_{B} T_{\alpha}}\left[v_{x}^{2}+\left(v_{y}-U_{\alpha}\right)^{2}+v_{z}^{2}\right]^{2}\right] .
\end{aligned}
$$


Fazendo alguns ajustes algébricos no argumento do exponencial, teremos,

$$
\begin{aligned}
f_{\alpha}(x, z, \vec{v})= & n_{0_{\alpha}}\left(\frac{m_{\alpha}}{2 \pi k_{B} T_{\alpha}}\right)^{3 / 2} \\
& \times \exp \left[\frac{-m_{\alpha}}{2 k_{B} T_{\alpha}}\left(v_{x}^{2}+\left(v_{y}-U_{\alpha}\right)^{2}+v_{z}^{2}\right)\right. \\
& \left.-\frac{q_{\alpha} \phi-q_{\alpha} U_{\alpha} A_{y}}{k_{B} T_{\alpha}}\right] .
\end{aligned}
$$

A equação (61) é a expressão final da forma analítica da função de distribuição que será utilizada no sistema de equações de Vlasov-Maxwell para se obter uma forma simplificada da equação de GS.

\section{Obtenção de uma Forma Simplificada da Equação de GS}

Substituindo $f_{j}(x, z, \vec{v})$ (representada em (61)) em (52), teremos:

$$
\begin{aligned}
\nabla \times & \vec{B} \\
= & \mu_{0} \sum_{\alpha} q_{\alpha} n_{0_{\alpha}}\left(\frac{m_{\alpha}}{2 \pi k_{B} T_{\alpha}}\right)^{\frac{3}{2}} \int_{v} \\
& \times\left[v_{y} \exp \left(\frac{-m_{\alpha}}{2 k_{B} T_{\alpha}}\left(v_{x}^{2}+\left(v_{y}-U_{\alpha}\right)^{2}+v_{z}^{2}\right)-\Theta\right)\right] \\
& \times d^{3} v
\end{aligned}
$$

para reduzir a expressão foi considerado que $\Theta=$ $\frac{q_{\alpha} \phi-q_{\alpha} U_{\alpha} A_{y}}{k_{B} T_{\alpha}}$.

Aplicando a propriedade $\exp [A+B]=\exp [A] \exp [B]$ no termo da direita de 62 com $U_{\alpha}$ constante e considerando que o potencial $A_{y}$ é constante por $y$ não variar, resta somente três integrais a serem resolvidas que ficam da forma:

$$
\begin{aligned}
& I_{1}=\int_{-\infty}^{\infty} \exp \left[-\frac{m_{\alpha} v_{x}^{2}}{2 k_{B} T_{\alpha}}\right] d v_{x} \\
& I_{2}=\int_{-\infty}^{\infty} v_{y} \exp \left[-\frac{m_{\alpha}\left(v_{y}-U_{\alpha}\right)^{2}}{2 k_{B} T_{\alpha}}\right] d v_{y}, \\
& I_{3}=\int_{-\infty}^{\infty} \exp \left[-\frac{m_{\alpha} v_{z}^{2}}{2 k_{B} T_{\alpha}}\right] d v_{z} .
\end{aligned}
$$

Elas podem ser resolvidas utilizando a propriedade matemática:

$$
\int_{-\infty}^{\infty} \exp \left(-\beta x^{2}\right) d x=\left(\frac{\pi}{\beta}\right)^{\frac{1}{2}} .
$$

Portanto as integrais ficarão da forma:

$$
\begin{aligned}
& I_{1}=I_{3}=\left(\frac{\pi}{\frac{m_{\alpha}}{2 k_{B} T_{\alpha}}}\right)^{\frac{1}{2}}=\left(\frac{2 \pi k_{B} T_{\alpha}}{m_{\alpha}}\right)^{\frac{1}{2}}, \\
& I_{2}=U_{\alpha}\left(\frac{\pi}{\frac{m_{\alpha}}{2 k_{B} T_{\alpha}}}\right)^{\frac{1}{2}}=U_{\alpha}\left(\frac{2 \pi k_{B} T_{\alpha}}{m_{\alpha}}\right)^{\frac{1}{2}} .
\end{aligned}
$$

logo, substituindo o resultado de $I_{1}, I_{2}$ e $I_{3}$ em $(62)$, a expressão de $J_{y}$ ficará:

$$
\begin{aligned}
J_{y}(x, z)= & \sum_{\alpha} n_{0_{\alpha}} q_{\alpha}\left(\frac{m_{\alpha}}{2 \pi k_{B} T_{\alpha}}\right)^{\frac{3}{2}}\left(\frac{2 \pi k_{B} T_{\alpha}}{m_{\alpha}}\right)^{\frac{1}{2}} \\
& \times\left(\frac{2 \pi k_{B} T_{\alpha}}{m_{\alpha}}\right)^{\frac{1}{2}}\left(U_{\alpha} \frac{2 \pi k_{B} T_{\alpha}}{m_{\alpha}}\right)^{\frac{1}{2}} \exp [-\Theta]
\end{aligned}
$$

e utilizando a propriedade matemática de multiplicação de potências de mesma base:

$$
\begin{aligned}
J_{y}(x, z)= & \sum_{\alpha} n_{0_{\alpha}} q_{\alpha} U_{\alpha}\left(\frac{m_{\alpha}}{2 \pi k_{B} T_{\alpha}}\right)^{\frac{3}{2}} \\
& \times\left(\frac{2 \pi k_{B} T_{\alpha}}{m_{\alpha}}\right)^{\frac{3}{2}} \exp [-\Theta] .
\end{aligned}
$$

Perceba que em 70 temos dois termos que podem ser simplificados, portanto:

$$
J_{y}(x, z)=\sum_{\alpha} n_{0_{\alpha}} q_{\alpha} U_{\alpha} \exp [-\Theta] .
$$

Assim a expressão $\nabla^{2} A_{y}=-\mu_{0} J_{y}$ ficará da seguinte forma:

$$
\nabla^{2} A_{y}=-\mu_{0} \sum_{\alpha} n_{0_{\alpha}} q_{\alpha} U_{\alpha} \exp [-\Theta]
$$

Para continuar com o raciocínio, substitui-se 61 em (51), isto é,

$$
\begin{aligned}
\nabla \cdot \vec{E}= & \frac{1}{\epsilon_{0}} \sum_{\alpha} q_{\alpha} n_{0_{\alpha}}\left(\frac{m_{\alpha}}{2 \pi k_{B} T_{\alpha}}\right)^{\frac{3}{2}} \int_{v} \\
& \times\left[\exp \left(\frac{-m_{\alpha}}{2 k_{B} T_{\alpha}}\left(v_{x}^{2}+\left(v_{y}-U_{\alpha}\right)^{2}+v_{z}^{2}\right)-\Theta\right)\right] \\
& \times d^{3} v .
\end{aligned}
$$

O resultado das integrais dentro do argumento do somatório é o mesmo apresentado para resolver 62, porém o resultado será:

$$
\rho(x, z)=\sum_{\alpha} q_{\alpha} n_{0_{\alpha}} \exp [-\Theta] .
$$

Expandindo o somatório para os dois tipos $\alpha$ de partículas, sendo $i$ de íons e $e$ de elétrons:

$$
\rho(x, z)=q_{i} n_{0_{i}} \exp \left[-\Theta_{i}\right]-q_{e} n_{0_{e}} \exp \left[-\Theta_{e}\right],
$$

em que foi atribuída carga negativa ao elétron.

Utilizando a propriedade de neutralidade macroscópica do plasma, fica evidente que $\rho(x, z)$ precisará se anular, mas isso somente será possível quando são satisfeitas as seguintes condições [28, 44]:

$$
\begin{aligned}
& \left|q_{i}\right|=\left|q_{e}\right|=q, \\
& n_{0_{i}}=n_{0_{e}}=n_{0}, \\
& \frac{U_{i}}{T_{i}}=-\frac{U_{e}}{T_{e}}, \\
& \phi(x, z)=0 .
\end{aligned}
$$


Logo, retornando na equação (72) com essas condições, segue que:

$$
\begin{aligned}
\nabla^{2} A_{y} & =-\mu_{0} q n_{0}\left(\frac{U_{i} k_{B} T_{i}}{k_{B} T_{i}}-\frac{U_{e} k_{B} T_{e}}{k_{B} T_{e}}\right) \exp \left[\frac{q U_{i}}{k_{B} T_{i}} A_{y}\right] \\
& =-2 \mu_{0} q n_{0} k_{B}\left(T_{i}+T_{e}\right) \frac{q U_{i}}{2 k_{B} T_{i}} \exp \left[\frac{q U_{i}}{k_{B} T_{i}} A_{y}\right] .
\end{aligned}
$$

Introduzindo duas constantes: $B_{0}^{2}=2 \mu_{0} n_{0} k_{B}\left(T_{i}+T_{e}\right)$ que é o campo magnético assintótico e $L=\frac{2 k_{B} T_{i}}{q U_{i} B_{0}}$, a equação 80 fica:

$$
\begin{aligned}
\nabla^{2} A_{y} & =-B_{0} \frac{1}{\frac{2 T_{i} k_{B}}{q U_{i} B_{0}}} \exp \left[\frac{q B_{0} U_{i}}{2 k_{B} T_{i}} \frac{2 A_{y}}{B_{0}}\right] \\
& =-\frac{B_{0}}{L} \exp \left[\frac{1}{L} \frac{2 A_{y}}{B_{0}}\right] \\
& =-\frac{B_{0}}{L} \exp [-2 \Psi] .
\end{aligned}
$$

sendo que $\Psi$ foi definido em (5). Portanto:

$$
\nabla^{2} A_{y}=\left(\frac{\partial^{2}}{\partial x^{2}}+\frac{\partial^{2}}{\partial z^{2}}\right)\left(-L B_{0} \Psi\right)=-\frac{B_{0}}{L} \exp [-2 \Psi] .
$$

Fazendo ajustes algébricos, teremos

$$
\nabla^{2} A_{y}=\left(\frac{\partial^{2}}{\partial\left(\frac{x}{L}\right)^{2}}+\frac{\partial^{2}}{\partial\left(\frac{z}{L}\right)^{2}}\right) \Psi=\exp [-2 \Psi]
$$

Introduzindo novas variáveis que não possuem dimensões: $\frac{x}{L}=X$ e $\frac{z}{L}=Z$, a equação ficará da forma apresentada em (7). Sendo que,

$$
J_{y}(x, z)=\frac{B_{0}}{L_{0} \mu_{0}} \exp (-2 \Psi) .
$$

A expressão $\nabla^{2} \Psi=\exp [-2 \Psi]$ é chamada de forma simplificada (ou específica) da equação de GS, como foi apresentada em (7). Se for encontrado o valor de $\Psi$ em (7), será possível determinar $J_{y}$ de (84). A solução analítica de (7) será abordada na próxima seção.

\section{Solução Analítica}

A Equação de GS (2), apresentada no começo deste artigo, não têm solução analítica. No entanto, pode-se resolver numericamente como um problema de Cauchy, sendo que a equação diferencial está sujeita a certas condições iniciais [20, 45, 46. Nos trabalhos de [20, 31 foram utilizados a solução analítica proposta por Fadeev e colaboradores 42, para criar um gráfico de contorno, que permitiu visualizar o erro percentual, útil na interpretação dos resultados da solução numérica.
Melhorar a solução numérica, permite aplicar o método de reconstrução de GS para recuperar várias estruturas em plasma espacial, a partir de dados, em particular de observações de nuvens magnéticas ou de tubos de fluxos magnéticos [20, 24, 25, 31, 45, 47,49. No entanto, apresentar o método de reconstrução de GS não forma parte do escopo deste trabalho, porque implicaria trabalhar com (2), sendo que nosso foco foi direcionado na equação (7). Novas soluções analíticas da equação (7) também poderão ser importantes para validar futuras melhorias na solução numérica e utilizá-las com condições iniciais em simulações MHD [50 59]. As soluções analíticas também são importantes para entender a coexistência entre os pontos neutro tipo-X, ilhas magnéticas e pontos singulares que aparecem, por exemplo, no modelo de Kan [29] e na solução analítica encontrada por 43 .

A equação (7) tem solução. Foi apresentada por Walker 60] em 1915, com aplicação na Astrofísica, dependendo da área do conhecimento [30, 33, 61,64], a solução está no contexto das funções complexas. Walker conseguiu uma solução usando uma função geradora $g(\zeta)$, sendo $\zeta=X+\iota Z$, que permite estudar uma variedade de soluções e modelos de lâminas de corrente. Vamos deixar explícita aqui a solução de Walker para fins didáticos:

$$
\exp [-2 \Psi]=\frac{4\left|g(\zeta)^{\prime}\right|^{2}}{\left(1+|g(\zeta)|^{2}\right)^{2}}
$$

Por exemplo, uma das soluções mais utilizada na literatura específica da área [20] é o modelo de Fadeev [42]. para obter o modelo de Fadeev, deve-se propor $g(\zeta)=$ $f_{p}+\sqrt{\left(1+f_{p}^{2}\right)} \exp (\iota \zeta)$. Após algum trabalho algébrico, encontra-se a solução:

$$
\Psi(X, Z)=\ln \left[f_{p} \cos (X)+\sqrt{1+f_{p}^{2}} \cosh (Z)\right],
$$

em que $f_{p}$ é uma constante que se utiliza para realizar manipulações no gráfico da solução.

A Figura 2 mostra a plotagem de $\vec{B}_{x z}=B_{x} \hat{i}+B_{z} \hat{k}$ (normalizado) em função das variáveis adimensionais $X$ e $Z$. Este tipo de figura permite visualizar a direção e sentido das linhas de campo magnético, permitindo identificar claramente as ilhas magnéticas e o ponto $X$ localizado na origem. A figura anterior foi superposta com o módulo de $J_{y}$ também normalizado entre 0 e 1 . A intensidade da densidade de corrente é caracterizada pela barra de cores, no qual ficará cada vez mais intensa a medida que a cor fica vermelha. Ela apresenta valor máximo no interior das ilhas magnéticas. Diminuindo o valor de $f_{p}$ para valores próximos de zero, o resultado será uma longa lâmina de corrente bidimensional no eixo $X$. 


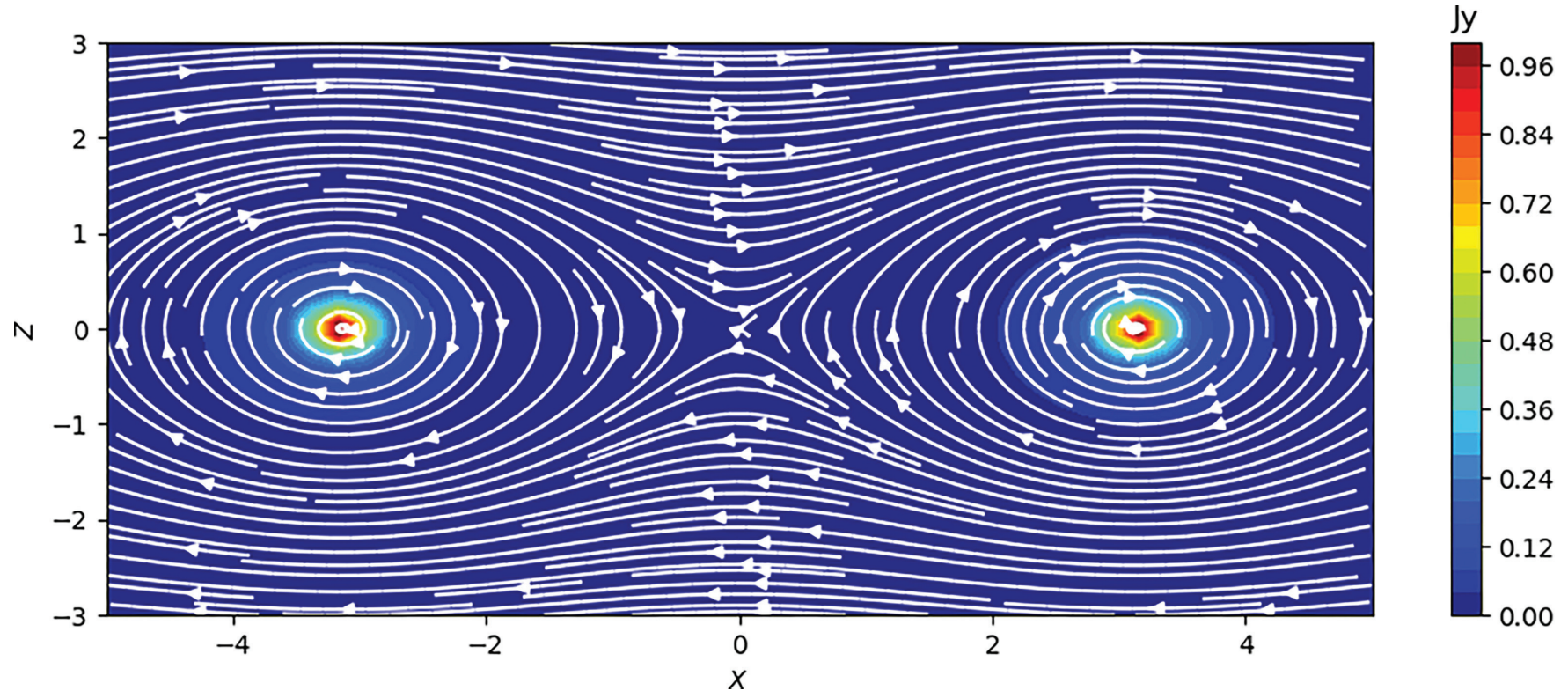

Figura 2: Configuração do campo magnético juntamente com a densidade de corrente normalizada em 2D no modelo de Fadeev. As linhas brancas representam a configuração do campo magnético com o sentido das linhas anti-paralelas. Note que, no ponto $(0,0)$ o valor do campo magnético tende a 0 , caracterizando assim o ponto $\mathbf{X}$ ou ponto neutro. Em torno dele, é possível ver as ilhas magnéticas caracterizadas pela geometria elíptica do campo. A densidade de corrente é caracterizada pela barra de cores, no qual ficará cada vez mais intensa a medida que a cor fica vermelha.

\section{Considerações Finais}

A equação de Grad-Shafranov é utilizada para se compreender processos físicos que ocorrem no geoespaço, por exemplo, na magnetosfera e em estruturas magnéticas do vento solar. A pesquisa aqui desenvolvida, foi elaborada visando uma construção sistemática e coerente com as teorias envolvidas nas áreas de física de plasmas, eletromagnetismo e a teoria Magnetohidrodinâmica (MHD). Esta é uma equação diferencial parcial (PDE) não-linear de segunda ordem importante para se fazer simulações de determinadas situações físicas no plasma geoespacial. Foi abordada e explicada a construção física-matemática utilizando teoria cinética em física de plasma para a obtenção de uma forma simplificada da equação de GS que, também, é uma forma simplificada da equação de Liouville. As soluções analíticas decorrentes da formula de Walker, dependendo do argumento da função geradora no qual são definidas, podem representar variedades de configurações topológicas de campo magnéticos, que em determinadas situações físicas, podem apresentar lâminas de corrente bidimensionais. Este artigo poderá servir como material de apoio para alunos de graduação e pós-graduação em cursos de física espacial, física de plasmas, eletromagnetismo e magnetohidrodinâmica.

\section{Agradecimentos}

M. Cristaldo Oliveira agradece ao PROSUC-CAPES pela bolsa de mestrado no curso de Física e Astrono- mia da UNIVAP. A. Ojeda González deseja agradecer ao Conselho Nacional de Desenvolvimento Científico e Tecnológico $(\mathrm{CNPq})$ pelo apoio financeiro (número de projeto 431396/2018-3). L. Nunes dos Santos agradece ao PROSUC-CAPES pela bolsa de doutorado no curso de Física e Astronomia da UNIVAP. Os autores agradecem o apoio da Profa. Nilvan Laurindo Sousa na correção do texto. Projeto vinculado ao Grupo de pesquisa do $\mathrm{CNPq}$ "Matemática Aplicada à Física Espacial".

\section{Referências}

[1] E.N. Parker, Astrophys. J. 128, 664 (1958).

[2] H. Alfven, Rev. Geophys 15, 271 (1977).

[3] M.J. Owens and R.J. Forsyth, Living Rev. Sol. Phys. 10, 5 (2013).

[4] E. Echer, Rev. Bras. Ens. Fis. 32, 2301-1 (2010).

[5] F.J.R. Simões Jr, E. Costa Jr, M.V. Alves and F.R. Cardoso, Rev. Bras. Ens. Fis. 33, 1310-1 (2011).

[6] P.R. Jauer, C. Wang, V.M. Souza, M.V. Alves, L.R. Alves, M. B. Pádua, J.P. Marchezi, L.A. Da Silva, Z. Liu, H. Liet al., Astrophys. J. 886, 1 (2019).

[7] D. Koga, W.D. Gonzalez, V.M. Souza, F.R. Cardoso, C. Wang and Z.K. Liu, J. Geophys. Res. Space Phy. 124, 8778 (2019)

[8] H. Alfvén, Nature 150, 405 (1942).

[9] H. Alfvén, Ark. Mat. Astr. Fys. 29, 1 (1943).

[10] E. Costa Jr., F.R. Cardoso, F.J.R. Simoes Jr. and M.V. Alves, Rev. Bras. Ens. Fis. 33, 2301-1 (2011).

[11] V.M. Souza, M.V.D. Silveira, D. Koga and P.R. Jauer, Rev. Bras. Ens. Fis. 38, e2301-1 (2016).

[12] N.F. Ness, J. Geophys. Res. 70, 2989 (1965). 
[13] R. G. Giovanelli, Nature 158, 81 (1946).

[14] J.W. Dungey, Phys. Rev. Lett. 6, 47 (1961).

[15] E.G. Zweibel and M. Yamada, Proc. Royal Soc. A 472, 20160479 (2016).

[16] P.P. Ferreira, S.M.V. Dias, F.R. Cardoso, D. Koga, V. M. Souza, L.E.V. Antunes, G. Farinas, B. M. CuadrosMelgar, W.D. Gonzalez and E. F. dos Santos, Rev. Bras. Ens. Fis. 39, e2307-1 (2017).

[17] W.D. Gonzalez, J.A. Joselyn, Y. Kamide, H.W. Kroehl, G. Rostoker, B.T. Tsurutani and V.M. Vasyliunas, J. Geophys. Res. 99, 5771 (1994).

[18] V. Klausner, M.O. Domingues, A.M. da Costa, A.R.R. Papa and A. Ojeda-Gonzalez, Adv. Space Res. 58, 2148 (2016).

[19] R. Schwenn, Living Rev. Sol. Phys. 3, 2 (2006).

[20] L.-N. Hau and B.U.Ö. Sonnerup, J. Geophys. Res. 104, 6899 (1999).

[21] B.U.O. Sonnerup, H. Hasegawa and G. Paschmann, Geophys. Res. Lett. 31, L11803 (2004).

[22] B.U.O. Sonnerup, H. Hasegawa, W.-L. Teh and L.-N. Hau, J. Geophys. Res. 111, 1 (2006).

[23] A. Isavnin, E.K.J. Kilpua and H.E.J. Koskinen, Sol. Phys. 273, 205 (2011).

[24] Q. Hu, Sci. China Earth Sci. 60, 1466 (2017).

[25] A. Ojeda-González, V. Klausner, O. Mendes, M.O. Domingues and A. Prestes, Sol Phys 292, 160 (2017).

[26] R.A.R. Oliveira, M.W.da S. Oliveira, A. Ojeda-González and V. De La Luz, Sol Phys 295, 45 (2020).

[27] A.V. Khrabrov and B.U.Ö. Sonnerup, ISSI Sci. Rep. S. 1, 221 (1998).

[28] J.R. Kan, J. Geophys. Res. 78, 3773 (1973).

[29] J.R. Kan, Planet. Space Sci. 27, 351 (1979).

[30] P.H. Yoon and A.T.Y. Lui, J. Geophys. Res. 110, A01202 (2005).

[31] A. Ojeda-González, M.O. Domingues, O. Mendes, M Kaibara and A. Prestes, Braz. J. Phys. 45, 493 (2015).

[32] A. Ojeda-González, in XXXVII Congresso Nacional de Matemática Aplicada e Computacional (São José dos Campos, 2018).

[33] A.V. Manankova, Ann. Geophys. 21, 2259 (2003).

[34] S.B. Zheng, A.J. Wootton and E.R. Solano, Phys. Plasmas 3, 1176 (1996).

[35] P.J. Mc Carthy, Phys. Plasmas 6, 3554 (1999).

[36] C.V. Atanasiu, S. Günter, K. Lackner and I.G. Miron, Phys. Plasmas 11, 3510 (2004).

[37] K. Schindler, Physics of Space Plasma Activity (Cambridge University Press, Cambridge, 2006), v. 1, p. 80.

[38] J.A. Bittencourt, Fundamentals of plasma physics. (Originally published by Pergamon Press, New York, (1986)), v. 1, p. 122.

[39] A. Ojeda-González, A. Prestes and A.N.L. Sousa, Braz. J. Phys. 46, 408 (2016).

[40] D. G. Crowdy, Int. J. Eng. Sci. 35, 141 (1997).

[41] D. Biskamp, Phys. Fluids 29, 1520 (1986).

[42] V.M. Fadeev, I.F. Kvabtskhava and N.N. Komarov, Nucl. Fusion 5, 202 (1965).

[43] A.N. Laurindo-Sousa, A. Ojeda-González, A. Prestes, V. Klausner and L. A. Caritá, Braz. J. Phys. 48, 67 (2018).

[44] E. G. Harris, II Nuovo Cimento 23, 115 (1962).
[45] B.U.Ö. Sonnerup and M. Guo, Geophys. Res. Lett. 23, 3679 (1996).

[46] Q. Hu and B.U.Ö. Sonnerup, J. Geophys. Res. 28, 467 (2001).

[47] Q. Hu, C.W. Smith, N.F. Ness and R.M. Skoug, J. Geophys. Res. Space Phy. 109, 3102 (2004).

[48] Q. Hu and B.U.O. Sonnerup, J. Geophys. Res. 107, 1142 (2002).

[49] Q. Hu and B.U.Ö. Sonnerup, J. Geophys. Res. 108, 1011 (2003).

[50] J. Birn and M. Hesse, J. Geophys. Res. 106, 3737 (2001).

[51] M. Hesse, J. Birn and M. Kuznetsova, J. Geophys. Res. 106, 3721 (2001).

[52] A. Otto, J. Geophys. Res. 106, 3751 (2001).

[53] M.A. Shay, J.F. Drake, B.N. Rogers and R.E. Denton, J. Geophys. Res. 106, 3759 (2001).

[54] Z.W. Ma and A. Bhattacharjee, J. Geophys. Res. 106, 3773 (2001)

[55] P.L. Pritchett, J. Geophys. Res. 106, 3783 (2001).

[56] M. M. Kuznetsova, M. Hesse and D. Winske, J. Geophys. Res. 106, 3799 (2001).

[57] U. Becker, T. Neukirch and K. Schindler, J. Geophys. Res. 106, 3811 (2001).

[58] K. Arzner and M. Scholer, J. Geophys. Res. 106, 3827 (2001).

[59] J.J. González-Avilés and F.S. Guzmán, IEEE Trans Plasma Sci. 46, 2378 (2018).

[60] G.W. Walker, Proc. Royal Soc. A. 91, 410 (1915).

[61] A.V. Manankova and M.I. Pudovkin, Geomagn. Aeron. 36, 426 (1996).

[62] A.V. Manankova and M.I. Pudovkin, Geomagn. Aeron. 39, 40 (1999).

[63] A.V. Manankova, M.I. Pudovkin and A.V. Runov, Geomagn. Aeron. 40, 430 (2000).

[64] D.B. Korovinskiy, D.I. Kubyshkina, V.S. Semenov, M.V. Kubyshkina, N.V. Erkaev and S.A. Kiehas, Ann. Geophys. 36, 641 (2018). 